\title{
Localized Flux Lines and the Bose Glass
}

\author{
Uwe C. Täuber \\ University of Oxford, Department of Physics - Theoretical Physics, 1 Keble Road, \\ Oxford OX1 3NP, U.K.
}

\begin{abstract}
Columnar defects provide effective pinning centers for magnetic flux lines in high $-T_{\mathrm{c}}$ superconductors. Utilizing a mapping of the statistical mechanics of directed lines to the quantum mechanics of two-dimensional bosons, one expects an entangled flux liquid phase at high temperatures, separated by a second-order localization transition from a low-temperature "Bose glass" phase with infinite tilt modulus. Recent decoration experiments have demonstrated that below the matching field the repulsive forces between the vortices may be sufficiently large to produce strong spatial correlations in the Bose glass. This is confirmed by numerical simulations, and a remarkably wide soft "Coulomb gap" at the chemical potential is found in the distribution of pinning energies. At low currents, the dominant transport mechanism in the Bose glass phase proceeds via the formation of double kinks between not necessarily adjacent columnar pins, similar to variable-range hopping in disordered semiconductors. The strong correlation effects originating in the longrange vortex interactions drastically reduce variable-range hopping transport.
\end{abstract}

\section{Pinning of Flux Lines to Columnar Defects}

The remarkably rich phase diagram of magnetic flux lines in high- $T_{\mathrm{c}}$ superconductors, especially when subject to point and / or extended disorder, has attracted considerable experimental and theoretical interest (for a recent review, see Ref. [1]). For the purpose of applying type-II superconductors in external magnetic fields, an effective vortex pinning mechanism is essential, in order to minimize dissipative losses caused by the Lorentz-force induced movement of flux lines across the sample. This issue becomes even more important for the high- $T_{\mathrm{c}}$ superconductors, because the strongly enhanced thermal fluctuations in these materials render the Abrikosov flux lattice unstable, and one therefore expects a first-order melting transition leading to a normal-conducting flux liquid phase with well below the upper critical field $H_{\mathrm{c}_{2}}(T)$, at least for ideally pure systems [2]. It is very difficult to pin such a vortex liquid consisting of fluctuating flexible lines by just intrinsic point disorder (e.g., oxygen vacancies); yet asymptotically a truly superconducting low-temperature "vortex glass" phase has been predicted [3].

Therefore, in addition to point defects, the influence of extended or correlated disorder, promising stronger pinning effects, on vortex transport properties has been considered. Experimentally, linear damage tracks have been produced in oxide superconductors by irradiation with high-energy ions. These 
columnar defects serve as strong pinning centers for the flux lines, thus significantly increasing the critical current and shifting the irreversibility line upwards (for references, see Ref. [1], Sec. IX B).

In a long-wavelength description in the spirit of the London approximation, one may consider the following model free energy for $N$ flux lines, defined by their trajectories $\mathbf{r}_{i}(z)$ as they traverse the sample, interacting with each other and with $N_{\mathrm{D}}$ columnar defects which are aligned along the magnetic-field direction $\hat{\mathbf{z}}[4]$, [5]:

$$
F_{N}=\int_{0}^{L} d z \sum_{i=1}^{N}\left\{\frac{\tilde{\epsilon}_{1}}{2}\left(\frac{\mathrm{d} \mathbf{r}_{i}(z)}{\mathrm{d} z}\right)^{2}+\frac{1}{2} \sum_{j \neq i}^{N} V\left[r_{i j}(z)\right]+\sum_{k=1}^{N_{\mathrm{D}}} V_{\mathrm{D}}\left[\mathbf{r}_{i}(z)-\mathbf{R}_{k}\right]\right\} .
$$

Here $r_{i j}(z)=\left|\mathbf{r}_{i}(z)-\mathbf{r}_{j}(z)\right|$, and $V(r)=2 \epsilon_{0} K_{0}(r / \lambda)$ denotes the repulsive vortex interaction; the modified Bessel function $\mathrm{K}_{0}(x) \propto-\ln x$ for $x \rightarrow 0$, and $\propto x^{-1 / 2} \mathrm{e}^{-x}$ for $x \rightarrow \infty$. Thus the London penetration depth $\lambda$ defines the interaction range and the energy scale $\epsilon_{0}=\left(\phi_{0} / 4 \pi \lambda\right)^{2}$. For low fields $B \leq \phi_{0} / \lambda^{2}$, i.e.: $\lambda \lesssim a_{0}=(4 / 3)^{1 / 4}\left(\phi_{0} / B\right)^{1 / 2}$, the vortex line tension is $\tilde{\epsilon}_{1} \approx \epsilon_{0}$ ( $a_{0}$ is the triangular lattice constant in the corresponding pure system). Finally, the pinning energy is a sum of $N_{\mathrm{D}} z$-independent potential wells $V_{\mathrm{D}}(\mathbf{r})$ with average spacing $d$ centered on the positions $\left\{\mathbf{R}_{k}\right\}$, whose diameters are typically $b_{0} \approx 100 \AA$, with a variation of $\delta b_{k} / b_{0} \approx 15 \%$, caused by the ionbeam dispersion. This induces some distribution $P$ of the pinning energies $U_{k}$, which may be determined from the formula $U_{k} \approx\left(\epsilon_{0} / 2\right) \ln \left[1+\left(b_{k} / \sqrt{2} \xi\right)^{2}\right]$, where $\xi$ denotes the coherence length [5]. The thermodynamic properties of the model (1) are to be obtained by evaluation of the grand-canonical partition function

$$
\mathcal{Z}_{\text {gr }}=\sum_{N=0}^{\infty} \frac{1}{N !} \mathrm{e}^{\mu N L / T} \int \prod_{i=1}^{N} \mathcal{D}\left[\mathbf{r}_{i}(z)\right] \mathrm{e}^{-F_{N}\left[\left\{\mathbf{r}_{i}(z)\right\}\right] / k_{\mathrm{B}} T},
$$

with the chemical potential $\mu=\left(H_{c_{1}}-H\right) \phi_{0} / 4 \pi$, which changes sign at the lower critical field $H_{c_{1}}=\phi_{0} \ln (\lambda / \xi) / 4 \pi \lambda^{2}$.

As for pure systems [2], one can map this statistical problem of directed flux lines interacting with columnar pins, onto the quantum mechanics of bosons in two dimensions subject to static point disorder via the identification of the vortex trajectories $\mathbf{r}_{i}(z)$ with the particle world lines in imaginary time [4], [5], [6]. In Table 1, the corresponding quantities for bosons and magnetic flux lines are listed (notice that the thermodynamic limit $L \rightarrow$ $\infty$ for the directed polymers corresponds to a zero-"temperature" quantum problem), and the ensuing phase diagram is sketched schematically in Fig. 1. At high temperatures, one thus finds an entangled liquid of unbound flux lines (corresponding to a boson superfluid), separated by a sharp second-order transition (see Ref. [7] and references therein) from a low-temperature phase of localized vortices. This Bose glass phase is characterized by an infinite tilt modulus $c_{44}$, and turns out to be stable over a certain range of tipping 
angles of $\mathbf{B}$ away from the $z$ direction [5], [8]. At least for $\lambda \ll d$, the theory also suggests a low-temperature Mott insulator phase, when $B=B_{\phi}$, i.e., the vortex density exactly matches that of the columnar pins. The Bose glass and the Mott insulator are distinct thermodynamic phases, for the latter should be characterized by infinite tilt and compressional moduli [5].

Table 1. Boson analogy applied to vortex transport.

\begin{tabular}{lccccccccc}
\hline Bosons & $m$ & $\hbar$ & $\beta \hbar$ & $V(r)$ & $n$ & $\mu$ & $\rho_{\mathrm{s}}$ & $\mathbf{E}$ & $\mathbf{j}$ \\
Vortices & $\tilde{\epsilon}_{1}$ & $T$ & $L$ & $2 \epsilon_{0} K_{0}(r / \lambda)$ & $B / \phi_{0}$ & $\left(H-H_{\mathrm{c}_{1}}\right) \phi_{0} / 4 \pi$ & $\rho^{2} c_{44}^{-1}$ & $\hat{\mathbf{z}} \times \mathbf{J} / c$ & $\mathcal{E}$ \\
\hline
\end{tabular}

Fig. 1. Schematic phase diagram for vortices interacting with columnar defects aligned along the magnetic-field direction.

The formal analogy with a boson superfluid can be further exploited to investigate the effect of disorder on density and current correlation functions in the flux liquid phase within the Bogoliubov approximation [9]. Thus one may compute the corresponding transport coefficients, e.g., the boson superfluid density $\rho_{\mathrm{s}}$ whose flux line analog is the inverse tilt modulus of the vortex array (see Table 1). For parallel columnar defects of strength $U_{0}$ one eventually finds [10]:

$$
c_{44}^{-1}(T) \approx\left(n \tilde{\epsilon}_{1}\right)^{-1}\left[1-\left(T_{\mathrm{BG}} / T\right)^{4}\right], \text { with } T_{\mathrm{BG}}(B) \approx T^{*}\left[\phi_{0} /(4 \pi \lambda)^{2} B\right]^{1 / 4}
$$

and $k_{\mathrm{B}} T^{*}=b\left(\tilde{\epsilon}_{1} U_{0}\right)^{1 / 2}$, in accord with estimates obtained in the Bose glass itself [5]. The critical behavior at the boson localization transition is not yet 
fully understood, although quantum Monte Carlo simulations [11] seem to support the scaling theory [7]. Direct simulations for the critical dynamics of flux lines near the Bose glass transition have also been performed [12].

\section{Structural Properties in the Bose Glass Phase}

Recently, the positions of flux lines and parallel columnar pins, aligned along the magnetic-field direction, were simultaneously measured in a BSCCO sample in the Bose glass phase at low magnetic fields using a combined chemical etching and magnetic decoration technique [13]. Figure 2 depicts the thus obtained positions of $N_{\mathrm{D}}$ columnar defects and $N$ flux lines in a two-dimensional cross section perpendicular to $\mathbf{B}$ in a case where $f=N / N_{\mathrm{D}}=B / B_{\phi} \approx 0.24$ and $\lambda \approx 0.45 a_{0}$. While the columnar defects are to a good approximation randomly distributed in space, the flux lines clearly form a highly correlated configuration; correspondingly, the two-dimensional vortex structure function $S(q)$ displays a distinct peak at wave vector $q \approx 2 \pi / a_{0}$, resembling an amorphous material (see Fig. 3). This correlated structure must obviously be the result of the mutual repulsive interactions $V_{\text {int }}$ between the vortices which dominate over any statistical fluctuations in the pinning potentials $\delta V_{\mathrm{D}}$; on the other hand, all the flux lines are attached to a defect, and therefore $V_{\mathrm{D}}>V_{\text {int }}[14]$.

Fig. 2. Positions of empty columnar defects (open circles), and pins occupied by flux lines (filled circles), as obtained from a decoration experiment $(f \approx 0.24)$.

In order to model this experimental situation, we can fortunately simplify the free energy (1) substantially: (i) As long as we limit ourselves to "low" temperatures (an estimate for BSCCO actually yields $T \lesssim T_{1}=0.9 T_{c}$ ) 
we can neglect thermal wandering of the flux lines, which is furthermore significantly suppressed by the confining line defects, we may drop the tiltenergy $\propto \tilde{\epsilon}_{1}$ and consider an effectively "classical" $\left(k_{\mathrm{B}} T \sim \hbar\right.$ in the boson analogy) two-dimensional system. (ii) For magnetic fields smaller than the crossover field $B^{*}(T)$ in Fig. 1, the vortices are sufficiently dilute to ensure that their repulsive interactions have no influence on the localization length, and in fact the "boson" statistics becomes irrelevant (for BSCCO, $B^{*}\left(T_{1}\right) \approx$ $\left.0.7 B_{\phi}\right)$. Having thus disposed of the entropic corrections, we may consider the following effective Hamiltonian and its grand-canonical counterpart [14], [15],

$$
H=\frac{1}{2} \sum_{i \neq j}^{N_{\mathrm{D}}} n_{i} n_{j} V\left(r_{i j}\right)+\sum_{i=1}^{N_{\mathrm{D}}} n_{i} t_{i}, \quad \tilde{H}=H-\mu \sum_{i=1}^{N_{\mathrm{D}}} n_{i},
$$

where $i=1, \ldots, N_{\mathrm{D}}$ denote the defect sites, randomly distributed in the $x$ $y$ plane, $n_{i}=0,1$ is the corresponding site occupation number, and the $t_{i}$ are random-site energies (originating in the varying pin diameters), whose distribution of mean zero and width $w \approx 0.1 l \epsilon_{0}$ we for simplicity choose to be flat: $P\left(t_{i}\right)=\Theta\left(w-\left|t_{i}\right|\right) / 2 w$.

This classical model is still far from trivial, however, due to the interplay of long-range interactions and disorder. Fortunately models of the form (4), albeit with a Coulomb potential replacing the screened logarithmic interaction $V(r)$, have been extensively studied in the context of disordered semiconductors, which allows us to adapt qualitative arguments and numerical simulation procedures from the literature (see, e.g., Refs. [16], [17]). Thus, in order to infer the (approximate) ground-state properties in the Bose glass phase as functions of the filling $f$ and interaction range $\lambda / d$ we employ a zero-temperature Monte Carlo algorithm that essentially minimizes the total energy (4) with respect to all possible one-vortex transfers; i.e., the ground state stability is checked by demanding that all single-particle hops from occupied sites $i$ to empty sites $j$ require an energy $\Delta_{i \rightarrow j}=\epsilon_{j}-\epsilon_{i}-V\left(r_{i j}\right)>0$, where $\epsilon_{i}=t_{i}+\sum_{j \neq i} n_{j} V\left(r_{i j}\right)$ are the interacting site energies. For $\lambda \gtrsim d$ and not too large $f \lesssim 0.6$ the resulting distribution of flux lines among the columnar pins closely resembles the experimental data in Fig. 2. More quantitatively, we can investigate the amorphous peak at $q a_{0} \approx 2 \pi$ in the vortex structure factor $S(\mathbf{q})=\sum_{i, j} \mathrm{e}^{\mathrm{i} \mathbf{q} \cdot\left(\mathbf{r}_{i}-\mathbf{r}_{j}\right)} / N$, averaged over all directions in reciprocal space. Whereas the dependence on the interaction range is rather weak as long as $\lambda \gtrsim d$, the peak in Fig. 3 vanishes when $f \gtrsim 0.4$; this reflects that at higher fillings the system has to increasingly accomodate with the underlying randomness, and therefore the correlations induced by the interactions disappear. Furthermore, a triangulation of the vortex positions shows that typically only about $50 \%$ of the sites are sixfold coordinated, and thus the structure formed by the flux lines rather resembles an amorphous state (justifying the name "Bose glass") rather than a weakly disordered triangular lattice [15]. 
Fig. 3. Dependence of the vortex structure factor peak on the filling $f$ for $\lambda / d=2$; solid: $f=0.1$, dot-dashed: $f=0.2$, dashed: $f=0.4$, and long-dashed: $f=0.8$.

\section{The Distribution of Pinning Energies}

The above Monte Carlo algorithm can also be employed to determine the distribution of pinning energies $g(\epsilon)$, with the vortex interactions taken into account. As in the boson picture this is nothing but the single-particle density of states, $g(\epsilon)$ is readily obtained by sampling the site energies $\epsilon_{i}$ for a number of disorder realizations and then performing a quenched average. Following the mean-field arguments of Efros and Shklovskii (1984) for a system with long-range repulsive interactions $V(r) \propto r^{-\sigma}$, with $0<\sigma<D$ (here, the space dimension is $D=2$ ), one expects that the density of states vanishes at the chemical potential according to a power law,

$$
g(\epsilon) \propto|\epsilon-\mu|^{s_{\text {eff }}},
$$

with a gap exponent $s_{\mathrm{MF}}=(D / \sigma)-1$. This means that as a consequence of the correlations induced by the long-range interactions, the probability of finding a low-energy empty site $(\epsilon \gtrsim \mu)$ for introducing an additional particle is greatly reduced, as is the density of states of high-energy filled sites $(\epsilon \lesssim \mu)$ for the corresponding process of removing a particle from the system. While the qualitative prediction (5) remains correct, the actual gap exponent is influenced by subtle fluctuation effects [17] and therefore differs from the mean-field predictions (for the currently most accurate results for Coulomb interactions, $\sigma=1$, see Ref. [18]).

Figure 4 depicts two characteristic examples for the distribution of pinning energies $g(\epsilon)$, as obtained from our simulations [15], and normalized according to $\int g(\epsilon) \mathrm{d} \epsilon=1 / d^{2}$. In the case of an infinite-range logarithmic potential, the Ewald summation method was used, and before taking the limit $\lambda \rightarrow \infty$, a term $\epsilon_{i}^{\prime}-\epsilon_{i}=-2 \pi f(\lambda / d)$ was subtracted from the site energies. The filled and empty states are separated by a wide soft gap centered at the 
Fig. 4. Normalized distribution of pinning energies $G(E)=2 \epsilon_{0} d^{2} g(\epsilon)$ as function of the single-particle energies $E=\epsilon / 2 \epsilon_{0}$, with (a) $\lambda \rightarrow \infty$, and $f=0.2$, (b) $\lambda / d=2$, and $f=0.4$. The location of $\mu$ is marked by the arrow.

chemical potential, with an effective gap exponent $s_{\text {eff }} \approx 3$ for low filling. Remarkably, this characteristic double-peak structure and the marked depletion of states persists even for finite-range repulsion with $\lambda \geq d$ (Fig. 4b), and although $g(\mu)$ does not strictly vanish any more, the distribution of pinning energies for $\epsilon \approx \mu$ can be fitted with a power law (5); e.g., $s_{\text {eff }} \approx 1.2$ for $\lambda / d=1$ and $f=0.1$. Upon increasing $f$, the gap closes quickly due to the stronger influence of the underlying randomness (compare Fig. 3), but for $f \lesssim 0.2$ the correlation effects only disappear when $\lambda<d$.

\section{Variable-Range Hopping Transport of Flux Lines}

An in-plane current $\mathbf{J} \perp \mathbf{B}$ induces a Lorentz force $\mathbf{f}_{\mathrm{L}}=\phi_{0} \hat{\mathbf{z}} \times \mathbf{J} / c$, acting on the vortices. Accordingly, a term $\delta F_{N}=-\int_{0}^{L} d z \sum_{i} \mathbf{f}_{\mathrm{L}} \cdot \mathbf{r}_{i}(z)$ has to be added to the free energy (1). In the spirit of the thermally assisted flux-flow (TAFF) model of vortex transport, we write the superconducting resistivity (i.e.: the conductivity in the boson representation) as $\rho=\mathcal{E} / J \approx \rho_{0} \exp \left[-U_{\mathrm{B}}(J) / T\right]$, where $\rho_{0}$ is a characteristic flux-flow resistivity, and $U_{\mathrm{B}}$ represents an effective barrier height. We shall see that in the Bose glass phase $U_{\mathrm{B}}(J)$ actually diverges as $J \rightarrow 0$,

$$
\left.\mathcal{E} \approx \rho_{0} J \exp \left[-E_{0} / k_{\mathrm{B}} T\right)\left(J_{0} / J\right)^{p_{\text {eff }}}\right] .
$$

Driven by an intermediate external current $J_{1}<J<J_{c}$, a flux line will start to leave its columnar pin by detaching a segment of length $z$ into the defect-free region, thereby forming a half-loop of transverse size $r$ (Fig. 5a). The free energy corresponding to this saddle-point configuration is readily estimated to be $\delta F_{1}(r, z) \approx \tilde{\epsilon}_{1} r^{2} / z+U_{0} z-f_{\mathrm{L}} r z$, and optimizing successively 
Fig. 5. Saddle-point configurations for vortex transport in the Bose glass phase. (a) Vortex half-loop. (b) Double-superkink in the variable-range hopping regime.

with respect to $z$ and $r$ one finds $z^{*} \approx r^{*} \sqrt{\tilde{\epsilon}_{1} / U_{0}} \approx c \sqrt{\tilde{\epsilon}_{1} U_{0}} / \phi_{0} J$. Hence the current-voltage characteristics assumes the form (6), with $E_{0}=E_{\mathrm{K}}=$ $d \sqrt{\tilde{\epsilon}_{1} U_{0}}, J_{0}=J_{1}=c U_{0} / \phi_{0} d$, and the effective transport exponent $p_{\text {eff }}=1$ in the vortex half-loop regime [5]. For $J \rightarrow J_{1}$, the flux line will extend a double kink of width $w_{\mathrm{K}}=d \sqrt{\tilde{\epsilon}_{1} / U_{0}}$ and energy $2 E_{\mathrm{K}}$ to an adjacent columnar defect, and therefore for $J<J_{1}$ neighboring pins must be taken into account.

Thus, at low currents one enters another regime, where the flux line emits a pair of kinks to a possibly distant defect which happens to provide a favorable pinning energy (Fig. 5b). This is the vortex analog of variable-range charge transport in disordered semiconductors (see Ref. [16] and references therein). The cost in free energy for such a configuration of transverse size $R$ and extension $Z$ along the magnetic-field direction will consist of the doublekink energy $2 E_{\mathrm{K}}(R)=2 E_{\mathrm{K}}(d) R / d$ stemming from the elastic term, and the difference in pinning energies of the highest-energy occupied site, $\epsilon_{i} \approx \mu$, and the empty site at distance $R$ with $\epsilon_{j}=\mu+\Delta(R)$, where the distribution of pinning energies enters through the condition $\int_{\mu}^{\mu+\Delta(R)} g(\epsilon) \mathrm{d} \epsilon=R^{-D}$. Minimizing the saddle-point free energy $\delta F_{\mathrm{SK}} \approx 2 E_{\mathrm{K}} R / d+Z \Delta(R)-f_{L} R Z$ in the regime where $g(\epsilon)$ has the form (5), one arrives at (6) with $E_{0}=2 E_{\mathrm{K}}$ and the effective transport exponent [15]

$$
p_{\mathrm{eff}}=\left(s_{\mathrm{eff}}+1\right) /\left(D+s_{\mathrm{eff}}+1\right) .
$$

For short-range interactions, $g(\mu)>0$, i.e., $s_{\text {eff }}=0$, and the Mott variable-range hopping exponent $p_{\mathrm{M}}=1 /(D+1)$ is recovered [5]. But as the interaction range $\lambda$ becomes larger than the average vortex separation $a_{0}=d / \sqrt{f}$, the emerging correlations reduce the phase space for singlevortex hopping drastically, and $p_{\text {eff }}<p_{\mathrm{M}}$; in addition, as the normalized distribution of pinning energies is broadened by the interactions, also the 
Fig. 6. $\log -\log$ plots (base 10) of the activation energy $U_{\mathrm{B}}(J) \propto R^{*}(J) / d$ for variable-range hopping vs the reduced current $j=J \phi_{0} d / 2 \epsilon_{0} c$ for $f=0.1$. Diamonds: $\lambda \rightarrow 0$, circles: $\lambda / d=1$, squares: $\lambda / d=2$, triangles: $\lambda / d=5$.

current scale $J_{0}$ in (6) is increased. The result of the minimization procedure using the numerical data for $g(\epsilon)$ in the case $f=0.1$ is shown in Fig. 6 as a function of the interaction range [15]. Assuming that flux line transport in the low-current regime is still dominated by single-vortex hops, this implies that long-range repulsive interactions reduce the resistivity by several orders of magnitudes with respect to the non-interacting situation. E.g., for $\log j \approx 1.5$ the exponent in (6) is about ten times smaller for $\lambda / d=5$ as compared to $\lambda \rightarrow 0$ (see Fig. 6), and hence $\rho$ is reduced by a factor of $\approx 10^{-5}$. In accord with the relation (7), the effective transport exponent assumes values upto $p_{\text {eff }} \approx 0.7$ for $\lambda \rightarrow \infty$ and $f=0.1$, while, e.g., for $\lambda / d=1$ and $f=0.1$ we find $p_{\text {eff }} \approx 0.55$. We remark that the former value for for the variable-range hopping exponent of logarithmically interacting particles in two dimensions is consistent with an analysis based on the very different assertion that actually collective many-particle hops yield the lowest energy barriers [19]. Finally, a recent experiment on a BSCCO sample with $f \approx 0.15$ and $\lambda / d \approx 1.6$ reported a measured $p_{\text {eff }} \approx 0.57$ in the variable-range hopping regime across about half a decade in the current-voltage characteristics [20]; this apparently agrees well with our results, although due caution needs to be applied for this comparison as in this experiment the magnetic field distribution was in fact very inhomogeneous. Yet further experiments should clearly be capable to test the above predictions quantitatively.

In summary, experiments have established that columnar defects provide very effective pinning centers for flux lines in high- $T_{c}$ superconductors, and demonstrated that in certain cases the repulsive vortex interactions lead to remarkable spatial correlations. As a consequence, a soft "Coulomb gap" emerges in the distribution of pinning energies, and vortex transport in the variable-range hopping regime should be drastically reduced. 
Acknowledgments. The work presented here was done in delightful collaboration with David Nelson, whose ideas and insights have been invaluable. I would also like to thank H. Dai and C.M. Lieber for fruitful cooperation and their sharing of data with us prior to publication. This research was supported by the Deutsche Forschungsgemeinschaft under Contract Ta. 177/1, and by the National Science Foundation, in part by the MRSEC Program through Grant DMR-9400396, and through Grant DMR-9417047. Financial support from a Royal Society Conference Grant and from the Engineering and Physical Sciences Research Council through Grant GR/J78327 is gratefully acknowledged.

\section{References}

[1] G. Blatter, M.V. Feigel'man, V.B. Geshkenbein, A.I. Larkin, and V.M. Vinokur, Rev. Mod. Phys. 66, 1125 (1994).

[2] D.R. Nelson, Phys. Rev. Lett. 60, 1973 (1988); D.R. Nelson and H.S. Seung, Phys. Rev. B 39, 9153 (1989).

[3] M.P.A. Fisher, Phys. Rev. Lett. 62, 1415 (1989); D.S. Fisher, M.P.A. Fisher, and D.A. Huse, Phys. Rev. B 43, 130 (1991).

[4] D.R. Nelson and V.M. Vinokur, Phys. Rev. Lett. 68, 2398 (1992).

[5] D.R. Nelson and V.M. Vinokur, Phys. Rev. B 48, 13060 (1993).

[6] I.F. Lyuksyutov, Europhys. Lett. 20, 273 (1992).

[7] M.P.A. Fisher, P.B. Weichman, G. Grinstein, and D.S. Fisher, Phys. Rev. B 40, 546 (1989).

[8] Further details on this "transverse Meissner effect" and vortex localization in transverse magnetic fields may be found in the contribution to this volume by D.R. Nelson.

[9] D.R. Nelson and P. Le Doussal, Phys. Rev. B 42, 10113 (1990).

[10] U.C. Täuber and D.R. Nelson, Preprint (1996).

[11] M. Wallin, E.S. Sørensen, S.M. Girvin, and A.P. Young, Phys. Rev. B 49, 12115 (1994).

[12] M. Wallin and S.M. Girvin, Phys. Rev. B 47, 14642 (1993).

[13] H. Dai, S. Yoon, J. Liu, R.C. Budhani, and C.M. Lieber, Science 265, 1552 (1994).

[14] U.C. Täuber, H. Dai, D.R. Nelson, and C.M. Lieber, Phys. Rev. Lett. 74, 5132 (1995).

[15] U.C. Täuber and D.R. Nelson, Phys. Rev. B 52, 16106 (1995).

[16] B.I. Shklovskii and A.L. Efros, Electronic properties of doped semiconductors, Springer-Verlag (New York, 1984).

[17] J.H. Davies, P.A. Lee, and T.M. Rice, Phys. Rev. Lett. 49, 758 (1982); Phys. Rev. B 29, 4260 (1984).

[18] A. Möbius, M. Richter, and B. Drittler, Phys. Rev. B 45, 11568 (1992).

[19] M.P.A. Fisher, T.A. Tokuyasu, and A.P. Young, Phys. Rev. Lett. 66, 2931 (1991).

[20] M. Konczykowski, N. Chikumoto, V.M. Vinokur, and M.V. Feigel'man, Phys. Rev. B 51, 3957 (1995). 